PROCEEDINGS OF THE

AMERICAN MATHEMATICAL SOCIETY

Volume 131, Number 8, Pages 2543-2547

S 0002-9939(02)06808-9

Article electronically published on November 27, 2002

\title{
A NOTE ON WEYL'S THEOREM FOR OPERATOR MATRICES
}

\author{
SLAVIŠA V. DJORDJEVIĆ AND YOUNG MIN HAN
}

(Communicated by Joseph A. Ball)

\begin{abstract}
When $A \in \mathcal{B}(X)$ and $B \in \mathcal{B}(Y)$ are given we denote by $M_{C}$ an operator acting on the Banach space $X \oplus Y$ of the form

$$
M_{C}=\left(\begin{array}{cc}
A & C \\
0 & B
\end{array}\right) \text {, where } C \in \mathcal{B}(Y, X) \text {. }
$$

In this note we examine the relation of Weyl's theorem for $A \oplus B$ and $M_{C}$ through local spectral theory.
\end{abstract}

\section{INTRODUCTION}

Throughout this note let $X$ and $Y$ be Banach spaces, let $\mathcal{B}(X, Y)$ denote the set of bounded linear operators from $X$ to $Y$, and abbreviate $\mathcal{B}(X, X)$ to $\mathcal{B}(X)$. If $T \in \mathcal{B}(X)$ we shall write $N(T)$ and $R(T)$ for the null space and range of $T$. Also, let $\alpha(T):=\operatorname{dim} N(T), \beta(T):=\operatorname{dim} X / R(T)$, and let $\sigma(T), \sigma_{d}(T), \sigma_{a}(T)$ and $\pi_{0}(T)$ denote the spectrum, defect spectrum, approximate point spectrum and point spectrum of $T$, respectively. An operator $T \in \mathcal{B}(X)$ is called upper semi-Fredholm if $R(T)$ is closed with finite dimensional null space and lower semi-Fredholm if $R(T)$ is closed with its range of finite co-dimension. If $T$ is both upper semi- and lower semi-Fredholm, we call it Fredholm. The index of a Fredholm operator $T$ is the integer $i(T):=\alpha(T)-\beta(T)$. An operator $T$ is called Weyl if it is Fredholm of index zero and is called Browder if it is Fredholm of "finite ascent and descent". The essential spectrum $\sigma_{e}(T)$, the Weyl spectrum $\omega(T)$ and the Browder spectrum $\sigma_{b}(T)$ of $T$ are defined by $([6,7])$ :

$$
\begin{aligned}
\sigma_{e}(T) & :=\{\lambda \in \mathbb{C}: T-\lambda \text { is not Fredholm }\}, \\
\omega(T) & :=\{\lambda \in \mathbb{C}: T-\lambda \text { is not Weyl }\}, \\
\sigma_{b}(T) & :=\{\lambda \in \mathbb{C}: T-\lambda \text { is not Browder }\},
\end{aligned}
$$

evidently

$$
\sigma_{e}(T) \subseteq \omega(T) \subseteq \sigma_{b}(T)=\sigma_{e}(T) \cup \operatorname{acc} \sigma(T),
$$

where we write acc $K$ for the accumulation points of $K \subseteq \mathbb{C}$.

If we write iso $K:=K \backslash$ acc $K$, then we let

$$
\pi_{00}(T):=\{\lambda \in \text { iso } \sigma(T): 0<\alpha(T-\lambda)<\infty\}
$$

denote the set of isolated eigenvalues of finite multiplicity.

Received by the editors January 21, 2002 and, in revised form, March 27, 2002.

2000 Mathematics Subject Classification. Primary 47A10, 47A55.

Key words and phrases. Upper triangular operator matrix, Weyl's theorem, single valued extension property. 
To say that "Weyl's theorem holds" for an operator $T \in \mathcal{B}(X)$ on a Banach space $X$ [7] is to claim that

$$
\sigma(T) \backslash \omega(T)=\pi_{00}(T) .
$$

The complement in the spectrum of the Weyl spectum is precisely the isolated points of the spectrum which are eigenvalues of finite multiplicity.

In this note we try to relate Weyl's theorem for an upper triangular operator matrix

$$
M_{C}=\left(\begin{array}{cc}
A & C \\
0 & B
\end{array}\right):\left(\begin{array}{l}
X \\
Y
\end{array}\right) \longrightarrow\left(\begin{array}{l}
X \\
Y
\end{array}\right)
$$

to the same thing for the diagonal matrix $M_{0}$, extending the work of Lee [11, 12 . on Hilbert spaces; our main tool is a local spectral theory.

\section{MAin RESUlts}

We begin with the following lemma.

Lemma 2.1. Let $A \in \mathcal{B}(X)$ and $B \in \mathcal{B}(Y)$. If $\left(\begin{array}{cc}A & 0 \\ 0 & B\end{array}\right)$ is Fredholm, then $M_{C}$ is Fredholm for every $C \in \mathcal{B}(Y, X)$. Hence, in particular, we have

$$
\sigma_{e}\left(M_{C}\right) \subseteq \sigma_{e}\left(\begin{array}{cc}
A & 0 \\
0 & B
\end{array}\right)=\sigma_{e}(A) \cup \sigma_{e}(B) .
$$

Proof. Suppose that $\left(\begin{array}{cc}A & 0 \\ 0 & B\end{array}\right)$ is Fredholm. Then $A$ and $B$ are both Fredholm and $i\left(\begin{array}{ll}A & 0 \\ 0 & B\end{array}\right)=i(A)+i(B)$. Observe that

$$
M_{C}=\left(\begin{array}{ll}
I & 0 \\
0 & B
\end{array}\right)\left(\begin{array}{ll}
I & C \\
0 & I
\end{array}\right)\left(\begin{array}{cc}
A & 0 \\
0 & I
\end{array}\right) .
$$

Since $\left(\begin{array}{ll}I & C \\ 0 & I\end{array}\right)$ is invertible for every $C \in \mathcal{B}(Y, X)$, and since $\left(\begin{array}{ll}I & 0 \\ 0 & B\end{array}\right)$ and $\left(\begin{array}{ll}A & 0 \\ 0 & I\end{array}\right)$ are both Fredholm, it follows that $M_{C}$ is Fredholm. The set inclusions in (2.1.1) are evident from the first assertion.

Lemma 2.2. Let $A \in \mathcal{B}(X)$ and $B \in \mathcal{B}(Y)$. Suppose that $\sigma_{*}(A) \subseteq \sigma_{*}\left(M_{C}\right)$ or $\sigma_{*}(B) \subseteq \sigma_{*}\left(M_{C}\right)$. Then $\sigma_{*}(A) \cup \sigma_{*}(B)=\sigma_{*}\left(M_{C}\right)$, where $\sigma_{*} \in\left\{\sigma, \sigma_{e}\right\}$.

Proof. Since $\sigma\left(M_{C}\right) \subseteq[\sigma(A) \cup \sigma(B)]$ by [5, Corollary 4], it is sufficient to show the opposite set inclusion. Suppose that $\sigma(A) \subseteq \sigma\left(M_{C}\right)$ or $\sigma(B) \subseteq \sigma\left(M_{C}\right)$, and assume to the contrary that $\sigma\left(M_{C}\right) \neq \sigma(A) \cup \sigma(B)$. Then there exists a $\lambda$ such that $\lambda \in[\sigma(A) \cup \sigma(B)] \backslash \sigma\left(M_{C}\right)$. It follows from 3, Theorem 5] that $\lambda \in \sigma(A) \cap \sigma(B)$, which implies that $\lambda \in \sigma\left(M_{C}\right)$, a contradiction. Therefore $\sigma(A) \cup \sigma(B)=\sigma\left(M_{C}\right)$.

Now we show that $\sigma_{e}(A) \cup \sigma_{e}(B)=\sigma_{e}\left(M_{C}\right)$. Since $\sigma_{e}\left(M_{C}\right) \subseteq\left[\sigma_{e}(A) \cup \sigma_{e}(B)\right]$ by Lemma 2.1, it is sufficient to show that $\left[\sigma_{e}(A) \cup \sigma_{e}(B)\right] \subseteq \sigma_{e}\left(M_{C}\right)$. Suppose that $\sigma_{e}(A) \subseteq \sigma_{e}\left(M_{C}\right)$ or $\sigma_{e}(B) \subseteq \sigma_{e}\left(M_{C}\right)$, and assume to the contrary that $\sigma_{e}\left(M_{C}\right) \neq$ $\sigma_{e}(A) \cup \sigma_{e}(B)$. Then there is a $\lambda$ such that $\lambda \in\left[\sigma_{e}(A) \cup \sigma_{e}(B)\right] \backslash \sigma_{e}\left(M_{C}\right)$. Observe that if $M_{C}$ is Fredholm, then $\left(\begin{array}{ll}I & 0 \\ 0 & B\end{array}\right)$ is lower semi-Fredholm and $\left(\begin{array}{cc}A & 0 \\ 0 & I\end{array}\right)$ is upper semi-Fredholm, respectively. Therefore $A$ is upper semi-Fredholm and $B$ is lower semi-Fredholm. From these observations, we notice that if $M_{C}$ is Fredholm, then $A$ is Fredholm if and only if $B$ is Fredholm. Hence $\lambda \in \sigma_{e}(A) \cap \sigma_{e}(B)$, a contradiction. Therefore $\sigma_{e}(A) \cup \sigma_{e}(B)=\sigma_{e}\left(M_{C}\right)$. This completes the proof. 
We say that $T \in \mathcal{B}(X)$ has the single valued extension property (SVEP) if for every open set $U$ of $\mathbb{C}$ the only analytic function $f: U \longrightarrow X$ which satisfies the equation

$$
(T-\lambda) f(\lambda)=0
$$

is the constant function $f \equiv 0$ on $U$. In this case, for $x \in X$ there is a maximal analytic function $f_{x}: U_{x} \rightarrow X$ satisfying $(T-\lambda) f_{x}(\lambda)=x$ on $U_{x}$. Set $\sigma_{T}(x):=$ $\mathbb{C} \backslash U_{x}$. Then $\sigma_{T}(x)$ is called the local spectrum at $x$. Recall from the local spectral theory of operators ([10]) that if $T \in \mathcal{B}(X)$ and $F$ is a closed subset of the complex plane $\mathbb{C}$, then we can define the spectral manifolds $\mathcal{X}_{T}(F)$ as follows:

$$
\begin{aligned}
\mathcal{X}_{T}(F):=\{x \in X: \text { there exists an analytic } X \text {-valued function } \\
\qquad f: \mathbb{C} \backslash F \longrightarrow X \text { such that }(T-\lambda) f(\lambda)=x\} .
\end{aligned}
$$

If $T$ has SVEP, then we have $\mathcal{X}_{T}(F)=\left\{x \in X: \sigma_{T}(x) \subseteq F\right\}$.

In general, $\sigma(A) \cup \sigma(B) \neq \sigma\left(M_{C}\right)$. Consider the following example: let $U \in \mathcal{B}\left(l_{2}\right)$ be the unilateral shift, $A=U$, and $B=U^{*}$. Then $\sigma\left(M_{0}\right)=\sigma(U) \cup \sigma\left(U^{*}\right)$ is the closed unit disk and $\sigma\left(M_{I-U U^{*}}\right)=\sigma\left(\begin{array}{cc}U & I-U U^{*} \\ 0 & U^{*}\end{array}\right)$ is the unit circle. Therefore $\sigma\left(M_{0}\right) \neq \sigma\left(M_{I-U U^{*}}\right)$. Notice that $U^{*}$ does not have SVEP. In [8], M. Houimdi and $\mathrm{H}$. Zguitti proved that if $B \in \mathcal{B}(Y)$ has SVEP, then $\sigma(A) \cup \sigma(B)=\sigma\left(M_{C}\right)$ for every $C \in \mathcal{B}(Y, X)$. Using Lemma 2.2 , we can extend this result as follows:

Theorem 2.3. Let $A \in \mathcal{B}(X)$ and $B \in \mathcal{B}(Y)$. Suppose that $A^{*}$ or $B$ has SVEP. Then for every $C \in \mathcal{B}(Y, X)$,

(1) $\sigma(A) \cup \sigma(B)=\sigma\left(M_{C}\right)$.

(2) $\sigma_{e}(A) \cup \sigma_{e}(B)=\sigma_{e}\left(M_{C}\right)$.

Proof. (1) Suppose that $A^{*}$ or $B$ has SVEP. It follows from 4, Corollary 7 and Theorem 2] that $\sigma_{a}(A)=\sigma(A)$ or $\sigma_{d}(B)=\sigma(B)$. Therefore we have that $\sigma(A) \subseteq$ $\sigma\left(M_{C}\right)$ or $\sigma(B) \subseteq \sigma\left(M_{C}\right)$ by [5, Theorem 2], and hence $\sigma(A) \cup \sigma(B)=\sigma\left(M_{C}\right)$ for every $C \in \mathcal{B}(Y, X)$ by Lemma 2.2 .

(2) Since $\sigma_{e}\left(M_{C}\right) \subseteq\left[\sigma_{e}(A) \cup \sigma_{e}(B)\right]$ by Lemma 2.1, it is sufficient to show that $\left[\sigma_{e}(A) \cup \sigma_{e}(B)\right] \subseteq \sigma_{e}\left(M_{C}\right)$. Without loss of generality, we assume that $0 \notin$ $\sigma_{e}\left(M_{C}\right)$. Then $M_{C}$ is Fredholm. Since $M_{C}$ is Fredholm, it follows from (2.1.1) that $A$ is upper semi-Fredholm and $B$ is lower semi-Fredholm, respectively. Suppose that $A^{*}$ has SVEP. Then it follows from [1, Theorem 2.8] that $i(A) \geq 0$. But $A$ is upper semi-Fredholm, hence $\alpha(A)<\infty$. Therefore $\beta(A)<\infty$, and so $A$ is Fredholm. Therefore $0 \notin \sigma_{e}(A)$, and so $\sigma_{e}(A) \subseteq \sigma_{e}\left(M_{C}\right)$. It follows from Lemma 2.2 that $\sigma_{e}(A) \cup \sigma_{e}(B)=\sigma_{e}\left(M_{C}\right)$ for every $C \in \mathcal{B}(Y, X)$. Now suppose that $B$ has SVEP. Then $i(B) \leq 0$ by [1, Theorem 2.6]. Since $B$ is lower semi-Fredholm, $\beta(B)<\infty$. Therefore $\alpha(B)<\infty$, and hence $B$ is Fredholm. So $0 \notin \sigma_{e}(B)$, and hence $\sigma_{e}(B) \subseteq \sigma_{e}\left(M_{C}\right)$. It follows from Lemma 2.2 that $\sigma_{e}(A) \cup \sigma_{e}(B)=\sigma_{e}\left(M_{C}\right)$ for every $C \in \mathcal{B}(Y, X)$.

If $\left(K_{n}\right)$ is a sequence of compact subsets of $\mathbb{C}$, then by the definition, its limit inferior is $\liminf K_{n}=\left\{\lambda \in \mathbb{C}:\right.$ there are $\lambda_{n} \in K_{n}$ with $\left.\lambda_{n} \longrightarrow \lambda\right\}$ and its limit superior is $\lim \sup K_{n}=\left\{\lambda \in \mathbb{C}:\right.$ there are $\lambda_{n_{k}} \in K_{n_{k}}$ with $\left.\lambda_{n_{k}} \longrightarrow \lambda\right\}$. If $\liminf K_{n}=\lim \sup K_{n}$, then $\lim K_{n}$ is defined by this common limit. A mapping $f$, defined on $\mathcal{B}(X)$, whose values are compact subsets of $\mathbb{C}$, is said to be upper (lower) semi-continuous at $T$, provided that if $T_{n} \longrightarrow T$ (in norm topology), then 
$\limsup f\left(T_{n}\right) \subseteq f(T)\left(f(T) \subseteq \liminf f\left(T_{n}\right)\right)$. If $f$ is both upper and lower semicontinuous at $T$, then it is said to be continuous at $T$ and in this case $\lim f\left(T_{n}\right)=$ $f(T)$.

Lemma 2.4. Let $A \in \mathcal{B}(X)$ and $B \in \mathcal{B}(Y)$. Then for every $C \in \mathcal{B}(Y, X)$

$$
\sigma_{b}\left(M_{C}\right) \subseteq \sigma_{b}\left(\begin{array}{cc}
A & 0 \\
0 & B
\end{array}\right)=\sigma_{b}(A) \cup \sigma_{b}(B) .
$$

Proof. Observe that

$$
M_{\frac{1}{n} C}=\left(\begin{array}{cc}
I & 0 \\
0 & n I
\end{array}\right)\left(\begin{array}{cc}
A & C \\
0 & B
\end{array}\right)\left(\begin{array}{cc}
I & 0 \\
0 & \frac{1}{n} I
\end{array}\right) \quad \text { for every } n \in \mathbb{N} .
$$

Since $\left\|\left(\begin{array}{cc}A & \frac{1}{n} C \\ 0 & B\end{array}\right)-\left(\begin{array}{cc}A & 0 \\ 0 & B\end{array}\right)\right\| \longrightarrow 0$ as $n \longrightarrow \infty$ and since $\sigma_{b}$ is upper semi-continuous by [13, Theorem 2], $\sigma_{b}\left(M_{C}\right) \subseteq \sigma_{b}\left(\begin{array}{cc}A & 0 \\ 0 & B\end{array}\right)$.

The second equality is obvious.

In general, "Weyl's theorem holds for $A \oplus B$ " does not imply "Weyl's theorem holds for $M_{C}$ " (see [12]). In spite of this situation, we have the following theorem. Recall that $T \in \mathcal{B}(X)$ is called isoloid if every isolated point of $\sigma(T)$ is an eigenvalue of $T$.

Theorem 2.5. Let $A \in \mathcal{B}(X)$ and $B \in \mathcal{B}(Y)$ both have SVEP. Suppose that $A$ and $B$ are isoloid and that $\mathcal{Y}_{B}(\{\lambda\})$ is finite dimensional for each $\lambda \in$ iso $\sigma(B)$. If Weyl's theorem holds for $A \oplus B$, then Weyl's theorem holds for $M_{C}$ for every $C \in \mathcal{B}(Y, X)$.

Proof. Since $A$ and $B$ both have SVEP, it follows from [8, Proposition 3.1] that $M_{C}$ has SVEP. Hence $\sigma\left(M_{C}\right) \backslash \omega\left(M_{C}\right) \subseteq \pi_{00}\left(M_{C}\right)$ by [2, Theorem 2.2]. Conversely, suppose that $\lambda \in \pi_{00}\left(M_{C}\right)$. Since $B$ has SVEP, $\sigma\left(M_{C}\right)=\sigma(A) \cup \sigma(B)$. Therefore $\lambda \in$ iso $(\sigma(A) \cup \sigma(B))$. Since $N(A-\lambda) \oplus\{0\} \subseteq N\left(M_{C}-\lambda\right)$ and $N\left(M_{C}-\lambda\right)$ is finite dimensional, $N(A-\lambda)$ is finite dimensional. Now we consider two cases:

Case 1: Suppose that $\lambda \in \sigma(A) \backslash \sigma(B)$. Since $N(A-\lambda)$ is finite dimensional and $B-\lambda$ is invertible, $N(A-\lambda) \oplus N(B-\lambda)$ is finite dimensional. On the other hand, since $\lambda \in$ iso $(\sigma(A) \cup \sigma(B)), \lambda \in$ iso $\sigma(A)$. Since $A$ is isoloid, $\operatorname{dim} N(A-\lambda)>0$. Therefore $\lambda \in \pi_{00}(A \oplus B)$. Since Weyl's theorem holds for $A \oplus B, \lambda \in \sigma(A \oplus B) \backslash$ $\omega(A \oplus B)$. But $A$ and $B$ have SVEP, hence $A-\lambda$ and $B-\lambda$ are both Browder by 2, Theorem 2.2]. It follows from Lemma 2.4 that $M_{C}-\lambda$ is Browder. Therefore $\lambda \in \sigma\left(M_{C}\right) \backslash \omega\left(M_{C}\right)$.

Case 2: Suppose that $\lambda \in \sigma(B)$. Since $\lambda \in$ iso $(\sigma(A) \cup \sigma(B)), \lambda \in$ iso $\sigma(B)$. It follows from [9, Theorem 3.1] that

$$
B=\left(\begin{array}{cc}
B_{1} & 0 \\
0 & B_{2}
\end{array}\right) \quad \text { on } \mathcal{Y}_{B}(\{\lambda\}) \oplus K(B-\lambda)
$$

with $\sigma\left(B_{1}\right)=\{\lambda\}$ and $\sigma\left(B_{2}\right)=\sigma(B) \backslash\{\lambda\}$.

Since $\mathcal{Y}_{B}(\{\lambda\})$ is finite dimensional, $B-\lambda$ is Weyl. Therefore $N(B-\lambda)$ is finite dimensional, and hence $N(A-\lambda) \oplus N(B-\lambda)$ is finite dimensional. On the other hand, since $B$ is isoloid, $\operatorname{dim} N(B-\lambda)>0$. Therefore $\lambda \in \pi_{00}(A \oplus B)$. Since Weyl's theorem holds for $A \oplus B, \lambda \in \sigma(A \oplus B) \backslash \omega(A \oplus B)$. But $A$ and $B$ have SVEP, hence $A-\lambda$ and $B-\lambda$ are both Browder by [2, Theorem 2.2]. It follows from Lemma 2.4 that $M_{C}-\lambda$ is Browder. Therefore $\lambda \in \sigma\left(M_{C}\right) \backslash \omega\left(M_{C}\right)$. This completes the proof. 


\section{ACKNOWLEDGEMENT}

The authors are grateful to the referee for several helpful suggestions concerning this paper.

\section{REFERENCES}

1. P. Aiena and O. Monsalve, Operators which do not have the single valued extension property, J. Math. Anal. Appl. 250 (2000), 435-448. MR 2001g:47005

2. S.V. Djordjević, B.P. Duggal and Y.M. Han, The single valued extension property and Weyl's theorem (preprint).

3. H.K. Du and J. Pan, Perturbation of spectrums of $2 \times 2$ operator matrices, Proc. Amer. Math. Soc. 121 (1994), 761-766. MR 94i:47004

4. J.K. Finch, The single valued extension property on a Banach space, Pacific J. Math. 58 (1975), 61-69. MR 51:11181

5. J.K. Han, H.Y. Lee and W.Y. Lee, Invertible completions of $2 \times 2$ upper triangular operator matrices, Proc. Amer. Math. Soc. 128 (2000), 119-123. MR 2000c:47003

6. R.E. Harte, Invertibility and Singularity for Bounded Linear Operators, Dekker, New York, 1988. MR 89d:47001

7. R.E. Harte and W.Y. Lee, Another note on Weyl's theorem, Trans. Amer. Math. Soc. 349 (1997), 2115-2124. MR 98j:47024

8. M. Houimdi and H. Zguitti, Propriétés spectrales locales d'une matrice carree des operateurs, Acta Math. Vietnamica 25 (2000), 137-144. MR 2001d:47011

9. J.J. Koliha, Isolated spectral points, Proc. Amer. Math. Soc. 124 (1996), 3417-3424. MR 97a:46057

10. K.B. Laursen and M.M. Neumann, An Intruduction to Local Spectra Theory, London Mathematical Society Monographs, New Series 20, Clarendon Press, Oxford 2000. MR 2001k:47002

11. W.Y. Lee, Weyl's theorem for operator matrices, Integral Equations and Operator Theory 32 (1998), 319-331. MR 99g:47023

12. W.Y. Lee, Weyl's spectra of operator matrices, Proc. Amer. Math. Soc. 129 (2001), 131-138. MR 2001f:47003

13. K.K. Oberai, Spectral mapping theorem for essential spectra, Rev. Roumaine Math. Pures Appl. 25 (1980), 365-373. MR 81j:47007

University of Niš, Faculty of Science, P.O. Box 91, 18000 Niš, Yugoslavia

E-mail address: slavdj@pmf.pmf.ni.ac.yu

Department of Mathematics, University of Iowa, 14 Maclean Hall, Iowa City, Iowa $52242-1419$

E-mail address: yhan@math.uiowa.edu 